\title{
A rare case of chronic nonbacterial osteomyelitis of the mandible in a young adult male
}

\author{
Yesel Kim¹, Jinhyuk Choi ${ }^{2}$, Hoi-In Jung ${ }^{3}$, and Jeong-Kui Kü* \\ ${ }^{1}$ Ph.D. Student, Department of Preventive Dentistry and Public Oral Health, Yonsei University, Seoul, Republic of Korea \\ ${ }^{2}$ Captain, Department of Forensic Medicine, Scientific Investigations Laboratory, Criminal Investigation Command, Ministry of National Defense, Seoul, \\ Republic of Korea \\ ${ }^{3}$ Assistant Professor, Department of Preventive Dentistry and Public Oral Health, Yonsei University, Seoul, Republic of Korea \\ ${ }^{4}$ Captain, Department of Oral and Maxillofacial Surgery, Section of Dentistry, Armed Forces Capital Dental Hospital, Armed Forces Medical Command, \\ Seongnam, Republic of Korea
}

\begin{abstract}
Chronic nonbacterial osteomyelitis (CNO), also known as chronic recurrent multifocal osteomyelitis, is a rare disease of unknown etiology mainly affecting girls with a mean age of 10 years. The disease is characterized by nonbacterial osteolytic lesions and swelling of the adjacent soft tissues and has nonstandard treatment protocols. Unifocal CNO typically involves the mandible. Mandibular CNO is difficult to differentiate from bacterial osteomyelitis because of its similar radiographic profile and clinical symptoms. Therefore, magnetic resonance imaging (MRI) should be considered if infectious osteomyelitis has not been diagnosed by laboratory examination. Our patient was a young adult man with mandibular CNO presented with atypical bone fragments on MRI. Clinical symptoms improved within 3 days after surgical intervention to remove the fragments and treatment with a nonsteroidal anti-inflammatory drug (NSAID). NSAID therapy was discontinued at postoperative day 20 , and the patient has experienced no recurrences at 5 months after surgery.
\end{abstract}

Key Words: Chronic recurrent multifocal osteomyelitis, Magnetic resonance imaging, Mandible, Oral surgery

@) This is an open-access article distributed under the terms of the Creative Commons Attribution Non-Commercial License (http://creativecommons.org/licenses/by-nc/4.0) which permits unrestricted noncommercial use, distribution, and reproduction in any medium, provided the original work is properly cited.

\section{INTRODUCTION}

Chronic nonbacterial osteomyelitis (CNO) is a rare inflammatory disease that is not associated with infection symptoms. The disease was introduced in 1972 as symmetric multifocal osteomyelitis [1]. CNO is also known as chronic recurrent multifocal osteomyelitis (CRMO) because of its recurrent characteristics on the multifocal region [2]. In addition, CRMO should be regarded as a childhood phe- notype of Synovitis-acne-pustulosis-hyperostosis-osteitis (SAPHO) syndrome [3,4]. Although the etiology of these diseases is unclear, it has been classified as an autoinflammatory disorder which is known as an immune system hyperactivation without high titers of autoreactive lymphocytes and autoantibodies [2]. Some studies reported that low-virulence microorganisms cause the disease, but others have suggested that mutations in genes encoding tumor necrosis factor (TNF)- $\alpha$ and interleukin-1 receptor antagonist

Received January 18, 2020; Revised February 10, 2020; Accepted February 10, 2020

${ }^{*}$ Corresponding author: Jeong-Kui Ku, Department of Oral and Maxillofacial Surgery, Section of Dentistry, Armed Forces Capital Dental Hospital, Armed Forces Medical Command, 81 Saemaeul-ro 177beon-gil, Bundang-gu, Seongnam 13574, Republic of Korea.

Tel: +82-31-725-6184, Fax: +82-31-706-0987, E-mail: kujk123@gmail.com

Copyright $\odot$ 2020, Oral Biology Research Institute 
could be the pathophysiological cause $[4,5]$. CNO should be differentiated from osteosarcoma, Ewing's sarcoma or Langerhans histiocytosis in a differential diagnosis [4]. The typical presentation of $\mathrm{CNO}$ is chronic nonbacterial inflammation, pain, and occasionally multifocal bone lesions with sclerotic bony margins [2].

$\mathrm{CNO}$ occurs predominantly in females with an average age of 10 years (1-17 years), and has been reported mainly on the long bone and clavicle with the pelvis and spine often being reported [6]. Approximately 1.5 to 3\% of multifocal CNO disease occurs in the mandible [6-8]. In particular, the mandible is the most commonly affected area as a single lesion [9]. Recurrence occurs an average of three months after stopping medication [10]. Most patients with a unifocal lesion of $\mathrm{CNO}$, who have a recurrence after drug treatment, develop multifocal lesions, and only $7 \%$ develop a unifocal lesion [8]. Few reports of adult CRMO have been documented [5]. In particular, no cases of mandibular unifocal lesion in young adult males have been reported [5].

For lesions of the jaw, a diagnosis is usually made using radiographs and computerized tomography $(\mathrm{CT})$ as the standard protocol. On the other hand, the radiographic aspect of mandibular $\mathrm{CNO}$ is difficult to differentiate from bacterial osteomyelitis, which is common mandibular disease and requires antibiotic treatment [11]. A mandibular $\mathrm{CNO}$ can be characterized from magnetic resonance imaging (MRI) as bone edema and high signal intensities in the masseter muscle [12,13]. Therefore, $\mathrm{CNO}$ is a diagnosis of exclusion by the clinical, imaging studies, and a culture negative bone biopsy results [14].

No standard treatment for $\mathrm{CNO}$ exists because there have been no randomized placebo-controlled trials. On the other hand, the use of nonsteroidal anti-inflammatory drugs (NSAIDs) for more than 6 months is recognized as a first choice. TNF blockers and bisphosphonates have also been used to treat $\mathrm{CNO}[15,16]$. CNO has been reported to improve with surgical intervention and NSAID and bisphosphonate because of the relatively easy access to the mandible compared to other bones (clavicle, vertebrae, pelvis, tibia, fibula, and femur) [16].

This paper reports a rare case of unifocal CNO of the mandible in a 20-year-old young adult male with an atypical bone fragment aspect, which was detected by MRI.
Successful results were obtained despite the short 20-day medication period after surgical intervention.

\section{CASE}

This study was conducted by IRB approval by the Institutinal Review Board at Armed Forces Capital Hospital (IRB no. AFCH-18-IRB-020). A 20-year-old male healthy soldier felt facial pain approximately two weeks prior. The patient visited the Emergency Room of the Armed Forces Capital Hospital and denied any facial trauma history. He complained of a suddenly occurred trismus of $10 \mathrm{~mm}$, facial pain, and swelling. No pathological findings, such as pericoronitis or periodontal disease, were found (Fig. 1A). $\mathrm{CT}$ revealed an osteolytic lesion and sclerotic changes on the right ramus, new bone formation, such as a periosteal reaction and enlarged mandibular foramen (Fig. 1B-D). A laboratory test performed at the time of admission did not indicate bacterial osteomyelitis (Table 1); therefore, MRI was performed to make a differential diagnosis. A high sig-

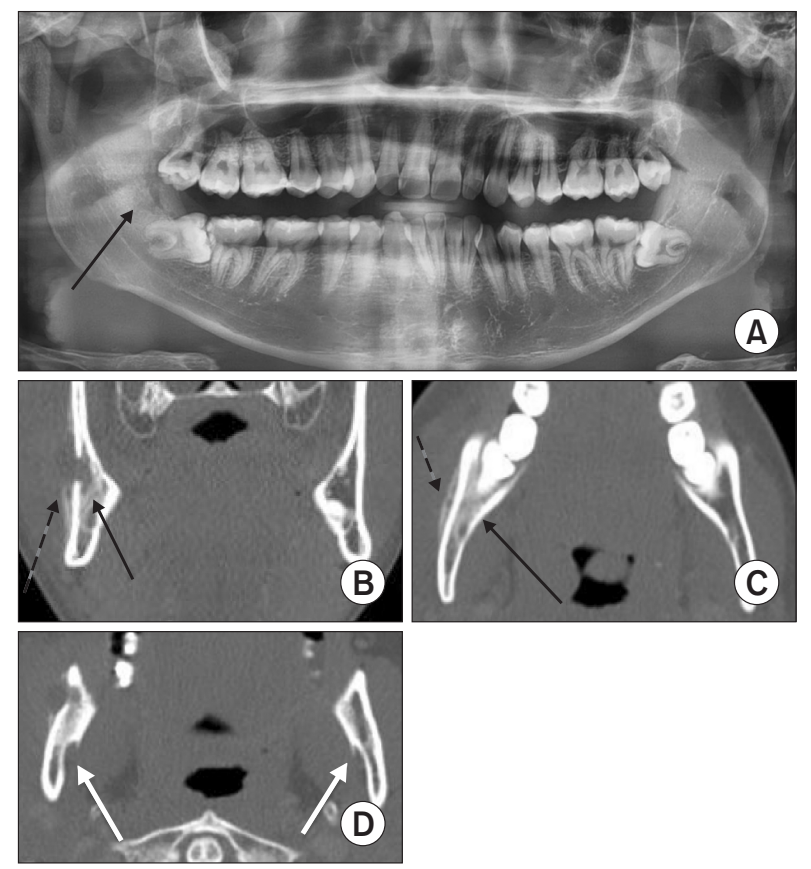

Fig. 1. Panoramic view and computerized tomography of mandibular chronic nonbacterial osteomyelitis. (A) Osteolytic lesion on the right mandibular ramus. (B, C) The black arrows showed an osteolytic lesion and sclerotic changes and the black dashed arrows showed new bone formation, such as a periosteal reaction. (D) Right mandibular foramen was greater than the opposite site (white arrows). 
Table 1. Laboratory test before and after operation

\begin{tabular}{|c|c|c|c|c|c|}
\hline Types of laboratory test & $\begin{array}{l}\text { Admission } \\
\text { day }\end{array}$ & $\begin{array}{c}\text { One hour } \\
\text { after operation }\end{array}$ & $\begin{array}{l}\text { Three days } \\
\text { after operation }\end{array}$ & $\begin{array}{c}\text { Seven days } \\
\text { after operation }\end{array}$ & $\begin{array}{c}\text { Normal } \\
\text { range }\end{array}$ \\
\hline Erythrocyte sedimentation rate $(\mathrm{mm} / \mathrm{hr})$ & 27 & 13 & 9 & 12 & $0-10$ \\
\hline C-reactive protein (mg/dL) & 0.4 & 0.2 & 0.2 & 0.1 & $0.5-1$ \\
\hline White blood cell $\left(10^{3} / \mu \mathrm{L}\right)$ & 11.8 & 11.3 & 11.0 & 11.0 & $4-10$ \\
\hline Neutrophil (\%) & 58.8 & 69.8 & 71.3 & 56.7 & $40-75$ \\
\hline Neutrophil (ABS, $\left.10^{3} / \mu \mathrm{L}\right)$ & 7.0 & 7.9 & 6.3 & 6.3 & $1.7-7.8$ \\
\hline Eosinophil (\%) & 2.5 & 2.4 & 0 & 4.1 & $<6$ \\
\hline Eosinophil (ABS, $\left.10^{3} / \mu \mathrm{L}\right)$ & 0.9 & 0.3 & 0 & 0.5 & $<0.5$ \\
\hline Body temperature $\left({ }^{\circ} \mathrm{C}\right)$ & 36.7 & 37.1 & 3.2 & 36.6 & $36.5-37.5$ \\
\hline
\end{tabular}

ABS, absolute.

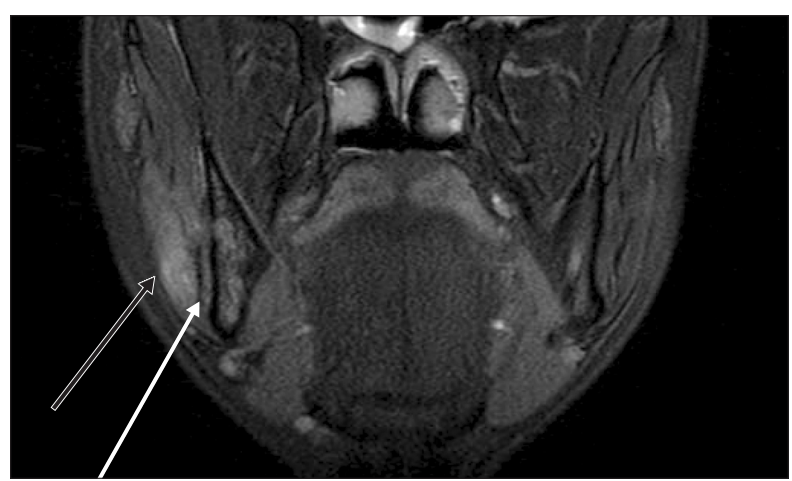

Fig. 2. T2-weighted magnetic resonance imaging with fat suppression of mandibular chronic nonbacterial osteomyelitis. High signal of the masseter muscle and ramus (black arrow), and bone fragment or cortical breakage (white arrow).

nal was observed on the T2-weighted with fat suppression MRI on the right masseter muscle and ramus, which are a typical presentation of $\mathrm{CNO}$, and an atypical presentation of bone fragments or cortical breakage was noted (Fig. 2). The symptoms did not improve even after 3 days of medication with cefoxitin sodium (Pacetin; JW pharmaceutical, Seoul, Korea) and acetamol hydrochloride (Denogan; AJU Pharm, Seoul, Korea) and surgical intervention was attempted. Local anesthesia (Lidocaine HCl; Huons, Seongnam, Korea) was injected into the right ramus area. An incision was made in the gingiva to the periosteum on the anterior ramus. An exploratory operation was performed on the osteolytic lesion of ramus through an osteotomy with a vulcanite bur. Only inflammatory tissue without pus or exudate discharge was noted and the pus culture could not be performed. The bone fragment was detected by MRI inside the masseter muscle and was removed with a blunt dissection (Fig. 3).

\section{Histologic findings}

In this case, inflamed granulation tissues infiltrating macrophages, neutrophils, plasma cells, and lymphocytes were observed without infectious microbial evidence in several foci (Fig. 3B, 4A). Broadly woven bone formations with an interconnecting structure and fibroblastic stroma were observed (Fig. 3D, 4B). Osteolysis was activated by osteoclasts around the woven bone (Fig. 3D, 4C). Fibrosis caused by chronic inflammation infiltrated into the surrounding skeletal muscle (Fig. 3D, 4D). Fibrosis was accompanied by lymphoplasmacytic infiltration. These histology features mean that subacute and chronic osteomyelitis appeared concurrently.

\section{Postoperative aspect}

The erythrocyte sedimentation rate (ESR) was decreased from 27 to 13 immediately after surgery. The remaining bone fragments were not observed by MRI taken on the day after surgery (Fig. 5A). ESR was decreased to nine and the symptoms of trismus, swelling, and pain were improved three days after surgery (Table 1).

Two weeks after surgery, a fever of more than $39^{\circ} \mathrm{C}$, itching sense, and macular skin rash occurred. A bone scan was taken to differentiate it from SAPHO syndrome (Fig. 5B). In this case, the skin lesion developed with fever, relative bradycardia, and eosinophilia (Fig. 5C, D). According to opinion of dermatology and infectious disease, the aspect 

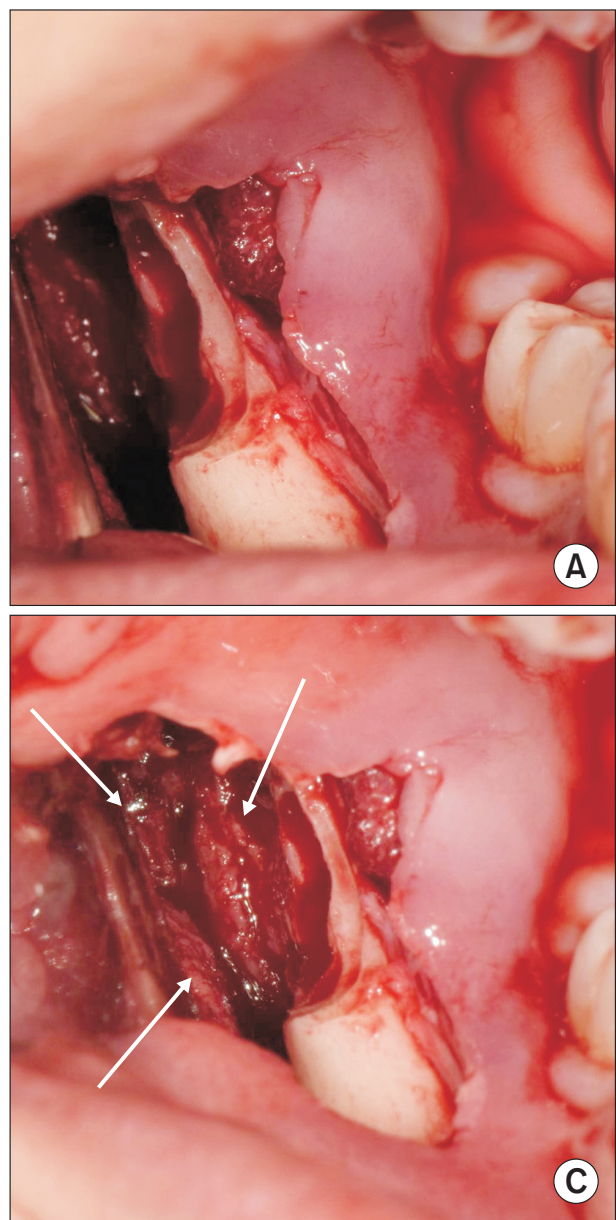

C
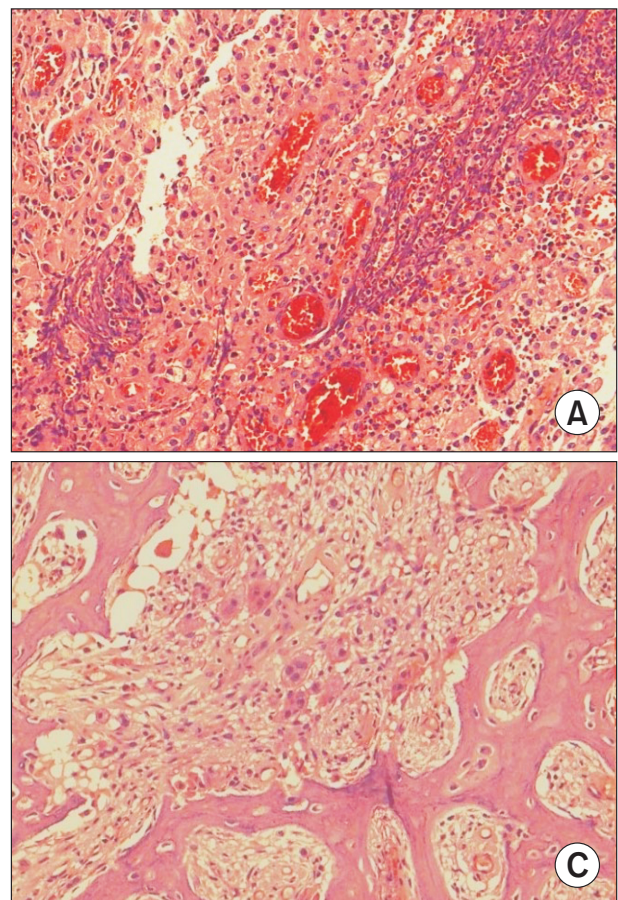
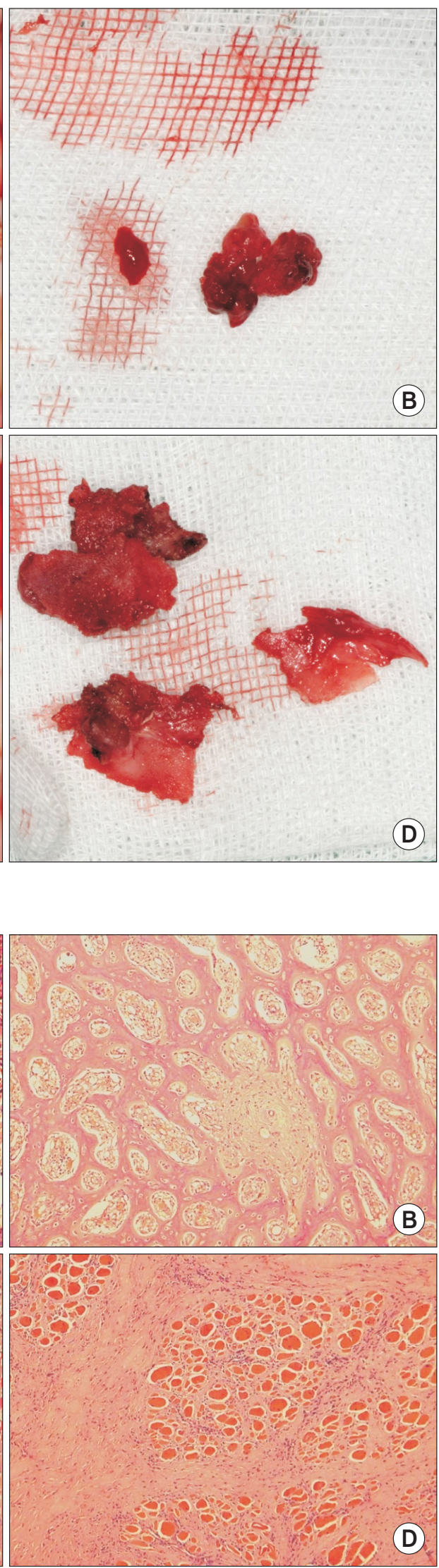

Fig. 3. Exploratory operation. (A) Access to the osteolytic lesion with anterior ramus osteotomy. (B) Inflammatory tissue from the osteolytic lesion. (C) The bone fragment revealed by magnetic resonance imaging. (D) Removed thin bone fragments with a blunt dissection.

Fig. 4. Histology findings of chronic nonbacterial osteomyelitis in this case (H\&E). (A) Inflamed granulation tissue infiltrating macrophages, neutrophils, plasma cells, and lymphocytes (magnification, $\times 200$ ). (B) Woven bone formation with an interconnecting structure and fibroblastic stroma (×100). (C) Activation of osteolysis around the woven bone $(\times 200)$. (D) Fibrosis infiltrating the surrounding skeletal muscle $(\times 100)$. 

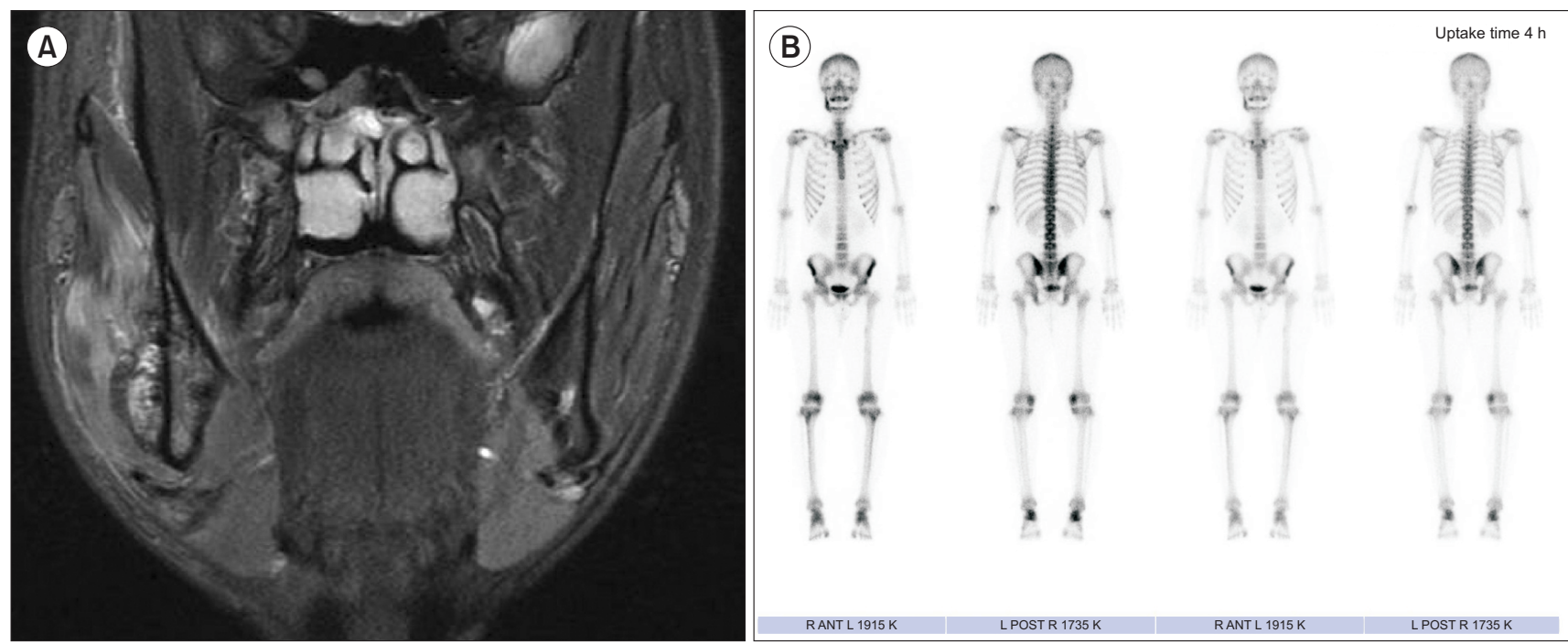

(C)

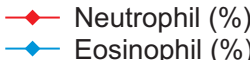

(D)
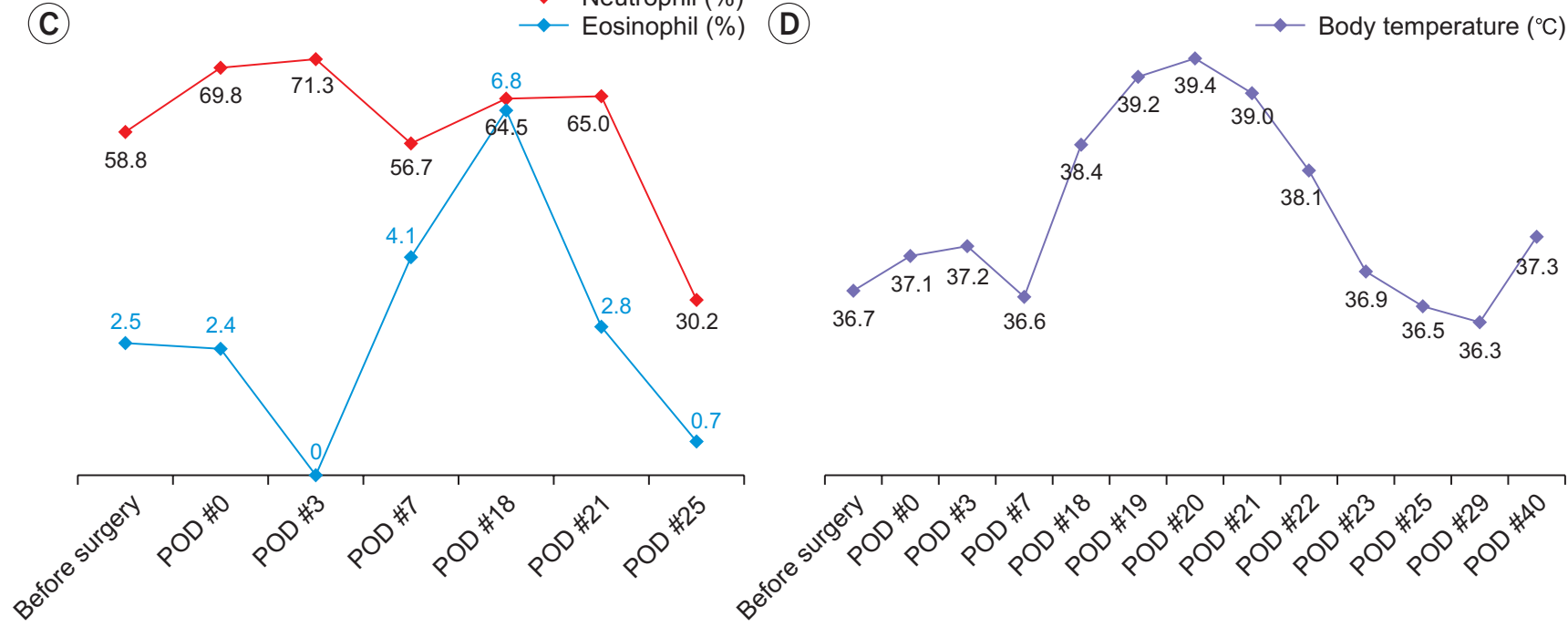

(E)

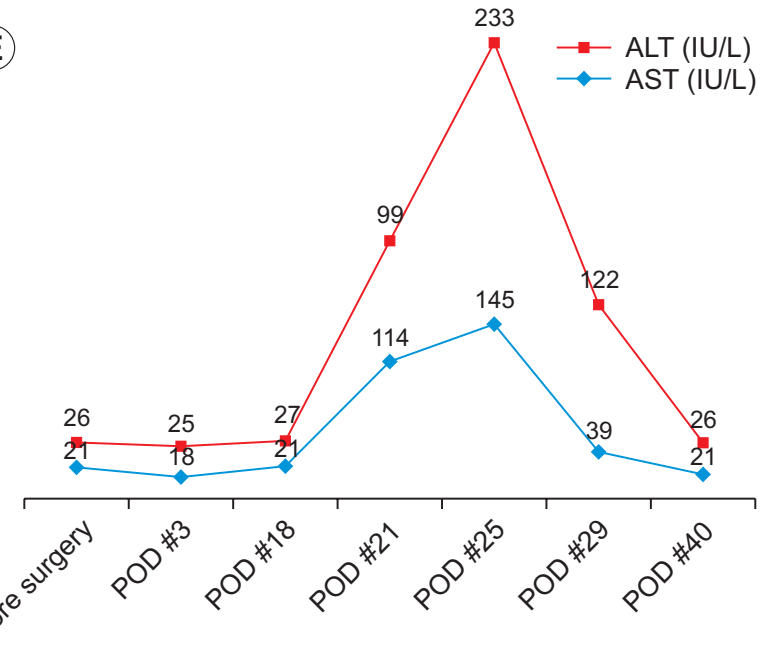

Fig. 5. Follow-up results within one month. (A) Magnetic resonance imaging taken the day after surgery. No remaining bone fragments were found. On the T2-weighted image with fat suppression, the range of high signal area was increased but the intensity of the signal, which had been spreading in the masseter decreased. (B) Bone scan at two weeks after surgery. Significantly increased radiotracer uptake in the right mandibular ramus and mild increased radiotracer uptake in the left mid foot at the Tc99m HDP 20mCi bone scan. (C) Eosinophil (\%) was increased before fever occurred and decreased after drug discontinuation. (D) Body temperature increased at 18 days after surgery and improved at three days after drug discontinuation. (E) Alanine aminotransferase (ALT) and aspartate aminotransferase (AST) were elevated after fever occurred (18 days after surgery) and improved at seven days after drug discontinuation. POD, postoperative day. 

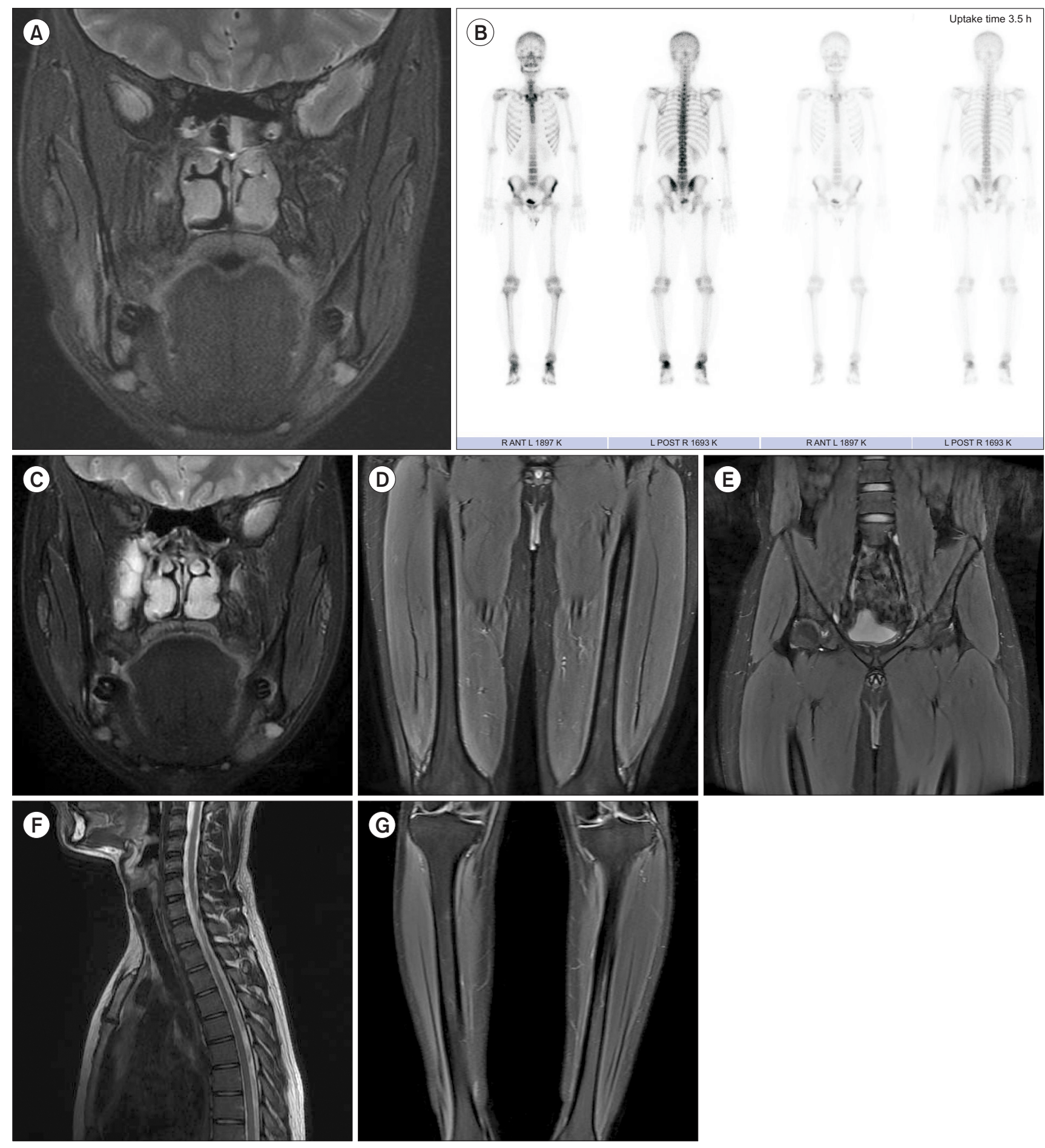

Fig. 6. Follow-up magnetic resonance imaging (MRI) and bone scan. (A) At one month after surgery. The signal intensity decreased on the right masseter muscle in the T2-weighted MRI image with fat suppression. (B) At four months after surgery. The intensity of radiotracer uptake decreased on the right mandibular ramus area, which had been increased significantly. The mild uptake of the elbow, knee and foot in the previous examination also improved. (C) Five months after surgery, the signal intensity of the master muscle was improved completely without the recurrence of chronic nonbacterial osteomyelitis (CNO) in the mandible. (D-G) There was no recurrence of CNO on other sites. (D) Femur, (E) pelvis, (F) spine, (G) tibia. 
of these symptoms was more similar to drug-induced fever than to SAPHO syndrome [17]. Therefore, antibiotics (cefoxitin sodium) and NSAIDs (acetamol hydrochloride) were discontinued and Desonide $0.5 \mathrm{mg} / \mathrm{mL}$ (Desowen lotion; Galderma, Seoul, Korea) was prescribed for the skin at 18 days after surgery. The fever and skin lesions were improved at five days after drug discontinuation (Fig. 5D). Alanine aminotransferase (ALT) and aspartate aminotransferase (AST) were elevated after the fever occurred and improved at 12 days after drug discontinuation (Fig. 5E).

The signal intensity decreased on the right masseter muscle in the follow up MRI at 32 days after surgery and the patient was discharged without clinical symptoms (Fig. 6A). A follow-up bone scan taken four months after surgery showed that the postoperative related uptake and remaining inflammatory processes were improved, and there was no recurrence of $\mathrm{CNO}$, including the other site (Fig. 6B). Five months after surgery, MRI showed no recurrence of $\mathrm{CNO}$ in the mandible and other sites (Fig. $6 \mathrm{C}-\mathrm{G}$ ). In addition, there were no CRMO findings of the pelvis and spine, including the elbow, knee, foot, and clavicle sites with slight uptake in the bone scan.

\section{DISCUSSION}

This study was reported a patient with $\mathrm{CNO}$ which was showed similar clinical and radiological aspect with bacterial osteomyelitis. He was revealed non-infectious result by laboratory test and typical aspects of CNO on MRI. Further, bone fragments-often reported in case of mandibular CNO [16] - were detected on MRI and reported histological findings.

In 1972, Giedion et al. [1] first described symmetric multifocal inflammatory bone lesions. In 2005, Girschick et al. [10] suggested the name, CNO, including CRMO, which also had a recurrent tendency because CRMO could be regarded as a severe form of $\mathrm{CNO}$. The disease has been reported in children aged 25 months to 17 years, and 1.5 to $3 \%$ of children diagnosed with multifocal CNO have lesions in the mandible $[7,8,18]$. In 2012, Borzutzky et al. [9] reported that $60 \%$ of unifocal $\mathrm{CNO}$ patients affected the mandible and $\mathrm{CNO}$ is rarely found in young adult males of 20 years. However, the prevalence of $\mathrm{CNO}$ could be un- derestimated because chronic non-suppurative mandibular osteomyelitis might be referred to as bacterial osteomyelitis with mild symptoms, primary chronic osteomyelitis, Garre osteomyelitis, juvenile mandibular chronic osteomyelitis, or diffuse sclerosing osteomyelitis [19].

This report shows that the $\mathrm{CT}$ image of the patient was suspected as osteomyelitis or sclerosing osteitis, but the laboratory results showing an absence of neutrophilia or $\mathrm{C}$-reactive protein (CRP) elevation indicated no bacterial infection. High signal intensities were observed diffusively on the MRI taken for a differential diagnosis, which are the same characteristics as CNO [12,13]. In addition, a 3.5x $2 \mathrm{~cm}$ fragment was noted as a cortical breakage aspect in MRI. Without taking MRI, it would be difficult to detect the fragment during operation because fully embedded in the masseter muscle. In 2016, Padwa et al. [16] reported devitalization of a jawbone fragment from $29 \%$ of $\mathrm{CNO}$ children (3 to 17 years) and there are no reports that the bone fragment was observed in other bones except the jaw. With regarding the fragments, histological, CT, or MRI analysis has not been clearly reported and the etiology of these bone fragments to be developed only in the jaw is still unknown. The histology findings of CNO can vary and be similar to infectious osteomyelitis in the acute and chronic stages [16]. However, histologic results of $\mathrm{CNO}$ are known as subacute or chronic inflammation, with a lymphocytic or mixed inflammatory infiltrate, and marrow fibrosis [14]. The histology examination of this patient revealed that the tissue at the site of osteolytic lesion to be chronic inflammatory tissue with infiltrating macrophages, neutrophils, plasma cells, and lymphocytes. Therefore, we could diagnosis this patient as CNO. In addition, the fragment was mixed with subacute and chronic osteomyelitis. With regarding the histological result, the bone fragment and the adjacent inflamed muscle could be related more to the progression of CNO symptoms than to the osteolytic lesion on the ramus.

The ESR, CRP, and serum TNF- $\alpha$ were reported to be significant for the diagnosis and follow-up of CNO [20]. In particular, ESR is related directly to the number of lesions detected radiologically in $\mathrm{CNO}$ [18]. In the present patient, ESR decreased from 27 to 13 immediately after removing the inflammatory bone lesion and bone fragment. At three days after surgery, ESR decreased to nine, and clinical 
symptoms, such as pain and swelling, were improved. ESR stabilized to 10 at one month after surgery. The MRI image of $\mathrm{T} 2$ with fat suppression on the day after surgery revealed a slight decrease in signal intensity at the masseter muscle, which was originally observed as high intensity. In addition, there was a significant decrease in the intensity on the masseter at five weeks after surgery on the MRI. This confirmed complete recovery with no recurrence on the mandible and other bones (pelvis, vertebrae, tibia, femur, and clavicle) on the follow-up MRI taken five months after surgery.

CNO has been treated with a variety of anti-inflammatory drugs as well as methotrexate, bisphosphonate, and anti-TNF- $\alpha$ agents as long-term medication for up to 12 years [21,22]. Beck et al. [18] reported that $43 \%$ of patients showed improved clinical symptoms with naproxen medication for six months. In terms of the side effects, Stern and Ferguson [14] suggested that indomethacin might be more effective than Naproxen in the recurrence case. Bisphosphonate has a positive effect in preventing the symptoms and progression of $\mathrm{CNO}$ [23]. In a retrospective cohort study in 2012, Borzutzky et al. [9] estimated the drug-specific response probabilities of CNO as 57\% for NSAID, 66\% for sulfasalazine, 91\% for methotrexate, and 95\% for corticosteroids. As CNO is a rare unknown disease, the standard medication therapy has not been established because there has not been any research with prospective randomized placebo-controlled trials for medication to treat $\mathrm{CNO}$. Although positive results were reported with only medication, such as anti-inflammatory agents and bisphosphonates, recent study suggest bone biopsy on mandibular CNO [2] . A successful result of mandibular $\mathrm{CNO}$ with surgical intervention and medication of NSAID and bisphosphonate was reported [16]. Fever, skin lesions of a morbilliform eruption, and elevation of AST and ALT occurred on the 20th day after surgery in this patient. Fortunately, the skin lesion of this patient manifested as a macular skin rash that was related to a drug-induced reaction not SAPHO syndrome [2]. Consequently, NSAID was administered for only 20 days because of drug fever and drug-induced liver injury. The medication period was shorter than that of conventional CNO studies, which reported an average of four to six months [21,22]. This patient showed a successful resolution of his clinical symptoms and a lower CRP (under 10) after 20 days of drug discontinuation (40 days after surgery). On the follow-up MRI, the inflammatory signal intensity, which had been spreading on the masseter muscle, also decreased significantly.

With regarding this patient, some auto genetic autoinflammatory bone syndromes could be considered such as Majeed syndrome, deficiency of interleukin-1 receptor antagonist (DIRA), Cherubism, and pyogenic arthritis, pyoderma gangrenosum, and acne (PAPA) syndrome $[2,14]$. Majeed syndrome is an autosomal recessive disorder with CRMO, congenital dyserythropoietic anemia, inflammatory dermatosis, and congenital dyserythropoietic anemia and inflammatory dermatosis [24]. DIRA is an autosomal recessive autoinflammatory disorder with extensive long bone involvement, a multifocal osteolytic pattern of disease, and marked periostitis [25]. Cherubism is an autosomal dominant autoinflammatory bone disorder with large multilocular, cystic lesion of the mandible and formation of fibrous tissue and atypical bone [26]. PAPA syndrome includes aseptic arthritis but there were few reports associated with posterior mandible [27]. Although these representative autoinflammatory diseases with non-infectious osteomyelitis differs from the symptoms of present patient [2], further studies could be considered for $\mathrm{CNO}$ patient including biomarkes such as antinuclear antibody, rheumatoid factor, and cyclic citrullinated peptide, gene and immunofluorescence analysis.

The clinical symptoms of this patient were resolved early without complications by removing the bone fragment and inflammatory tissue on the osteolytic lesion. The symptoms of mandibular $\mathrm{CNO}$ were improved at three days of NSAID and antibiotic medication and surgical intervention. Despite the insufficient studies of $\mathrm{CNO}$, many studies reported a recurrence rate of $80 \%$ or more at three to 90 months after symptoms were improved with medication [9,10,21]. A successful result was achieved with no recurrence of mandible or clavicle, vertebrae, pelvis, tibia, fibula, and femur on MRI taken 5 months after surgery with only 20 days of medication.

In conclusion, mandibular $\mathrm{CNO}$ is difficult to differentiate from bacterial osteomyelitis because of its similar radiological and clinical symptoms. Therefore, MRI should be con- 
sidered if infectious osteomyelitis has not been diagnosed on a laboratory examination.

In mandibular $\mathrm{CNO}$, atypical bone fragments were observed on a MRI image, which have not been reported in other bones and not detected on the CT image. This patient was treated successfully with surgical intervention of removing the fragment and short-term NSAID medication.

\section{CONFLICTS OF INTEREST}

The authors declare that they have no competing interests.

\section{ORCID}

\author{
Yesel Kim \\ https://orcid.org/0000-0002-0002-9757 \\ Jinhyuk Choi \\ https://orcid.org/0000-0002- 0316-5973 \\ Hoi-In Jung \\ https://orcid.org/0000-0002-1978-6926 \\ Jeong-Kui Ku \\ https://orcid.org/0000-0003-1192-7066
}

\section{REFERENCES}

1. Giedion A, Holthusen W, Masel LF, Vischer D. [Subacute and chronic "symmetrical" osteomyelitis]. Ann Radiol (Paris) 1972;15:329-342. Multiple languages.

2. Kim SM, Lee SK. Chronic non-bacterial osteomyelitis in the jaw. J Korean Assoc Oral Maxillofac Surg 2019;45:68-75. doi: 10.5125/jkaoms.2019.45.2.68.

3. Sato H, Wada Y, Hasegawa E, Nozawa Y, Nakatsue T, Ito T, Kuroda T, Saeki T, Umezu H, Suzuki Y, Nakano M, Narita I. Adult-onset chronic recurrent multifocal osteomyelitis with high intensity of muscles detected by magnetic resonance imaging, successfully controlled with tocilizumab. Intern Med 2017;56:2353-2360. doi: 10.2169/internalmedicine.8473-16.

4. Monsour PA, Dalton JB. Chronic recurrent multifocal osteomyelitis involving the mandible: case reports and review of the literature. Dentomaxillofac Radiol 2010;39:184-190. doi: $10.1259 / \mathrm{dmfr} / 23060413$.

5. Hong CW, Hsiao EC, Horvai AE, Link TM. Chronic recurrent multifocal osteomyelitis with an atypical presentation in an adult man. Skeletal Radiol 2015;44:1359-1364. doi: 10.1007/s00256-015-2130-8.

6. Chen Z, Cheng L, Feng G. Bone inflammation and chronic recurrent multifocal osteomyelitis. Eur Rev Med Pharmacol Sci 2018;22:1380-1386. doi: 10.26355/eurrev_201803_14482.

7. Jansson A, Renner ED, Ramser J, Mayer A, Haban M, Meindl A, Grote V, Diebold J, Jansson V, Schneider K, Belohradsky BH. Classification of non-bacterial osteitis: retrospective study of clinical, immunological and genetic aspects in 89 patients. Rheumatology (Oxford) 2007;46:154-160. doi: 10.1093/rheumatology/kel190.

8. Wipff J, Adamsbaum C, Kahan A, Job-Deslandre C. Chronic recurrent multifocal osteomyelitis. Joint Bone Spine 2011;78:555-560. doi: 10.1016/j.jbspin.2011.02.010.

9. Borzutzky A, Stern S, Reiff A, Zurakowski D, Steinberg EA, Dedeoglu F, Sundel RP. Pediatric chronic nonbacterial osteomyelitis. Pediatrics 2012;130:e1190-e1197. doi: 10.1542/peds.2011-3788.

10. Girschick HJ, Raab P, Surbaum S, Trusen A, Kirschner S, Schneider P, Papadopoulos T, Müller-Hermelink HK, Lipsky PE. Chronic non-bacterial osteomyelitis in children. Ann Rheum Dis 2005;64:279-285. doi: 10.1136/ $\operatorname{ard} .2004 .023838$.

11. Taddio A, Ferrara G, Insalaco A, Pardeo M, Gregori M, Finetti M, Pastore S, Tommasini A, Ventura A, Gattorno M. Dealing with chronic non-bacterial osteomyelitis: a practical approach. Pediatr Rheumatol Online J 2017;15:87. doi: 10.1186/s12969-017-0216-7.

12. Fritz J, Tzaribatchev N, Claussen CD, Carrino JA, Horger MS. Chronic recurrent multifocal osteomyelitis: comparison of whole-body MR imaging with radiography and correlation with clinical and laboratory data. Radiology 2009;252:842-851. doi: 10.1148/radiol.2523081335.

13. Karchevsky M, Schweitzer ME, Morrison WB, Parellada JA. MRI findings of septic arthritis and associated osteomyelitis in adults. AJR Am J Roentgenol 2004;182:119-122. doi: 10.2214/ajr.182.1.1820119.

14. Stern SM, Ferguson PJ. Autoinflammatory bone diseases. Rheum Dis Clin North Am 2013;39:735-749. doi: 10.1016/ j.rdc.2013.05.002.

15. Compeyrot-Lacassagne S, Rosenberg AM, Babyn P, Laxer RM. Pamidronate treatment of chronic noninfectious inflammatory lesions of the mandible in children. J Rheumatol 2007;34:1585-1589.

16. Padwa BL, Dentino K, Robson CD, Woo SB, Kurek K, Resnick CM. Pediatric chronic nonbacterial osteomyelitis of the jaw: clinical, radiographic, and histopathologic features. J Oral Maxillofac Surg 2016;74:2393-2402. doi: 10.1016/j.joms.2016.05.021.

17. Fauci AS, Braunwald E, Kasper DL, Hauser SL, Longo DL, Jameson JL, Loscalzo J. Harrison's principles of internal medicine. 17th ed. New York: McGraw-Hill Medical; 2008.

18. Beck C, Morbach H, Beer M, Stenzel M, Tappe D, Gattenlöhner S, Hofmann U, Raab P, Girschick HJ. Chronic nonbacterial osteomyelitis in childhood: prospective follow- 
up during the first year of anti-inflammatory treatment. Arthritis Res Ther 2010;12:R74. doi: 10.1186/ar2992.

19. van Merkesteyn JPR, Groot RH, Bras J, McCarroll RS, Bakker DJ. Diffuse sclerosing osteomyelitis of the mandible: a new concept of its etiology. Oral Surg Oral Med Oral Pathol 1990;70:414-419. doi: 10.1016/0030-4220(90)90200-C.

20. Jansson A, Renner ED, Ramser J, Mayer A, Haban M, Meindl A, Grote V, Diebold J, Jansson V, Schneider K, Belohradsky BH. Classification of non-bacterial osteitis: retrospective study of clinical, immunological and genetic aspects in 89 patients. Rheumatology (Oxford) 2007;46:154-160. doi: 10.1093/rheumatology/kel190.

21. Kaiser D, Bolt I, Hofer M, Relly C, Berthet G, Bolz D, Saurenmann T. Chronic nonbacterial osteomyelitis in children: a retrospective multicenter study. Pediatr Rheumatol Online J 2015;13:25. doi: 10.1186/s12969-015-0023-y.

22. Nelson H, Sebrén A. Medical treatment of chronic noninfectious osteomyelitis in the jaws. A systematic review [Thesis]. Malmö: Malmö University Faculty of Odontology; 2018.

23. Montonen M, Li TF, Lukinmaa PL, Sakai E, Hukkanen M, Sukura A, Konttinen YT. RANKL and cathepsin K in diffuse sclerosing osteomyelitis of the mandible. J Oral Pathol Med 2006;35:620-625. doi: 10.1111/j.1600-0714.2006.00454.x.

24. Majeed HA, Al-Tarawna M, El-Shanti H, Kamel B, Al-
Khalaileh F. The syndrome of chronic recurrent multifocal osteomyelitis and congenital dyserythropoietic anaemia. Report of a new family and a review. Eur J Pediatr 2001;160:705-710. doi: 10.1007/s004310100799.

25. Aksentijevich I, Masters SL, Ferguson PJ, Dancey P, Frenkel J, van Royen-Kerkhoff A, Laxer R, Tedgård U, Cowen EW, Pham TH, Booty M, Estes JD, Sandler NG, Plass N, Stone DL, Turner ML, Hill S, Butman JA, Schneider R, Babyn P, El-Shanti HI, Pope E, Barron K, Bing X, Laurence A, Lee CC, Chapelle D, Clarke GI, Ohson K, Nicholson M, Gadina M, Yang B, Korman BD, Gregersen PK, van Hagen PM, Hak AE, Huizing M, Rahman P, Douek DC, Remmers EF, Kastner DL, Goldbach-Mansky R. An autoinflammatory disease with deficiency of the interleukin-1-receptor antagonist. N Engl J Med 2009;360:2426-2437. doi: 10.1056/NEJMoa0807865.

26. Tiziani V, Reichenberger E, Buzzo CL, Niazi S, Fukai N, Stiller M, Peters H, Salzano FM, Raposo do Amaral CM, Olsen BR. The gene for cherubism maps to chromosome 4p16. Am J Hum Genet 1999;65:158-166. doi: $10.1086 / 302456$.

27. Yeon HB, Lindor NM, Seidman JG, Seidman CE. Pyogenic arthritis, pyoderma gangrenosum, and acne syndrome maps to chromosome 15q. Am J Hum Genet 2000;66:14431448. doi: 10.1086/302866. 